\title{
Réévaluation des apports moyens de matières en suspension de l'Arve au Rhône
}

\author{
Marina Launay ${ }^{1}$, Jérôme Le Coz ${ }^{1, *}$, Seydina Diouf ${ }^{2}$, Benoît Camenen ${ }^{1}$, Fabien Thollet $^{1}$ et \\ Marina Coquery ${ }^{1}$ \\ ${ }^{1}$ Irstea, UR RiverLy, centre de Lyon-Villeurbanne, 5, rue de la Doua, CS 20244, 69625 Villeurbanne, France \\ 2 SIG, Case postale 2777, 1211 Genève 2 , Suisse
}

Reçu le 30 mai 2018 / Accepté le 26 février 2019

Résumé - L'Arve est le principal contributeur au flux de matières en suspension (MES) du Rhône entre le lac Léman et Lyon. Une évaluation fiable des apports moyens de MES de l'Arve est cruciale pour la connaissance et la gestion des flux sédimentaires sur ce secteur du Rhône et à travers ses aménagements hydroélectriques. Cette étude vise à réévaluer les flux moyens de l'Arve en suspension fine, en croisant les données historiques avec les flux de MES de référence pour les années 2012 à 2017 issus du suivi turbidimétrique en continu à la station de Genève Bout-du-Monde de l'Observatoire des sédiments du Rhône. Ce jeu de données a permis d'évaluer les deux méthodes de calcul des flux mises en œuvre par le Service hydrologique national suisse pour les périodes avec des mesures discontinues des MES : la méthode des débits classés et la courbe de tarage sédimentaire à deux segments. En combinant au mieux ces méthodes, le flux moyen interannuel entre 1965 et 2017 est estimé à $0,68 \mathrm{Mt} / \mathrm{an}$ avec un écart-type de $0,29 \mathrm{Mt} / \mathrm{an}$. Cette valeur est cohérente avec le flux moyen mesuré par la station turbidimétrique entre 2012 et $2017(0,63 \mathrm{Mt})$ et avec un suivi historique pour l'année $1890(0,71 \mathrm{Mt})$; en revanche, elle est nettement inférieure à l'estimation précédemment proposée par l'étude globale Rhône (2000), comprise entre 1,0 et 3,6 Mt/an, qui inclut sans doute la suspension graduée de sables et limons grossiers, très mal connue. À partir de relevés bathymétriques et granulométrique des dépôts, nous avons estimé que la retenue de Verbois intercepte approximativement $0,1 \mathrm{Mt}$ /an de particules grossières issues de l'Arve et environ la moitié des apports de MES.

Mots clés : flux / matières en suspension (MES) / Arve / Rhône / Observatoire des sédiments du Rhône (OSR)

\begin{abstract}
Re-estimation of mean suspended solid delivery of the Arve River to the Rhône River. The Arve River is the main contributor of the suspended particulate matter (SPM) flux to the Rhône River between Lake Geneva and the city of Lyon. A reliable evaluation of the Arve River average delivery is crucial for the knowledge and management of sedimentary fluxes in this section of the Rhône and through its hydroelectric installations. This study aims to re-evaluate the average fine suspension flux of the Arve by crossing historical data with reference SPM fluxes for the years 2012 to 2017 derived from continuous turbidity monitoring at the Geneva-Bout-du-Monde station of the Rhône Sediment Observatory. This dataset allowed evaluating the two flux calculation methods implemented by the Swiss National Hydrological Service for periods with discontinuous SPM measurements: the flow exceedance method and the two-segment sediment rating curve. Using the best combination of these methods, the average interannual flux between 1965 and 2017 was estimated at $0.68 \mathrm{Mt} / \mathrm{yr}$ with a standard deviation of $0.29 \mathrm{Mt} / \mathrm{yr}$. This value is consistent with the average flux measured by the turbidity station between 2012 and 2017 $(0.63 \mathrm{Mt})$ and with historical monitoring for the year $1890(0.71 \mathrm{Mt})$; nonetheless, it is well below the previous estimate proposed by the Étude globale Rhône (2000) report: between 1.0 and 3.6 Mt/year, which probably included the graded suspension of coarse silt and sand, very poorly known. From bathymetric and grainsize surveys of the deposits, it is estimated that the Verbois reservoir captures approximately $0.1 \mathrm{Mt} /$ year of coarse particles from the Arve River, and about half of its SPM inputs.
\end{abstract}

Keywords: flux / suspended particulate matter (SPM) / Arve / Rhône / Rhône sediment observatory (OSR)

\footnotetext{
*Correspondance : jerome.lecoz@irstea.fr
} 


\section{Introduction}

L'Arve est le principal contributeur au flux de matières en suspension (MES) du Haut-Rhône français (Sogréah, 2003 ; Launay, 2014). Une évaluation fiable de ses apports moyens est donc cruciale pour connaître les flux de contaminants particulaires et pour optimiser la gestion des flux sédimentaires à travers les aménagements hydro-électriques du Rhône en aval de l'Arve. La sédimentation est un enjeu particulièrement important pour la retenue de Verbois, dont l'envasement augmente fortement le risque d'inondation dans les bas quartiers de Genève, ainsi que pour la retenue de Génissiat, qui retient une partie du flux sédimentaire lors des opérations de chasse des barrages suisses (Guertault et al., 2018). Cette question est au cœur de la recherche de modalités de gestion des flux alternatives aux chasses périodiques. En effet, ces opérations de gestion sédimentaire engendrent de forts niveaux de concentration en MES sur de courtes périodes (typiquement 1 à 2 semaines); une régulation précise des flux et concentrations permet d'éviter de potentiels dommages écologiques et d'importantes retombées socio-économiques négatives.

La présente étude a pour objectif de réévaluer les flux moyens de l'Arve en sédiments en suspension, en distinguant clairement la suspension fine homogène de la suspension graduée de sables. Pour éviter toute confusion, seul le premier type de suspension sera désigné par MES, le second étant appelé suspension de sables, même s'il peut s'agir de sables très fins et de limons grossiers. Cette étude croise les données historiques disponibles avec les flux de MES de référence récents pour les années 2012 à 2017 issus du suivi turbidimétrique en continu à la station de Genève Bout-du-Monde, mise en place par l'Observatoire des sédiments du Rhône (OSR) (Le Bescond et al., 2018). Ce jeu de données a permis d'évaluer les deux méthodes de calcul des flux mises en œuvre par le Service hydrologique national suisse pour les périodes avec des mesures discontinues des MES : la méthode des débits classés et la courbe de tarage sédimentaire à deux segments. En combinant au mieux ces méthodes, le flux de MES moyen interannuel entre 1965 et 2017 a été estimé et comparé avec le flux moyen mesuré par la station turbidimétrique entre 2012 et 2017, avec le suivi historique pour l'année 1890 par Baëff (1891), et avec l'estimation précédemment proposée par l'étude globale Rhône (2000). À partir de relevés bathymétriques et granulométrique des dépôts interceptés dans la retenue de Verbois, nous avons aussi estimé les apports de l'Arve en suspension graduée de sables et limons grossiers, qui restent très mal connus.

\section{Matériel et méthodes}

\subsection{Site d'étude}

L'Arve à Genève (station $\mathrm{OFEV}^{1}$ Bout-du-Monde ${ }^{2}$ ) a un bassin versant de $1976 \mathrm{~km}^{2}$. Avec un débit spécifique de $40 \mathrm{~L} / \mathrm{s} /$ $\mathrm{km}^{2}$ et un module (débit moyen interannuel) de $75 \mathrm{~m}^{3} / \mathrm{s}$ (moyenne des débits horaires de 1965 à 2017), il contribue à

\footnotetext{
${ }^{1}$ Office fédéral de l'environnement (Suisse).

${ }^{2} \mathrm{http}: / /$ www.hydrodaten.admin.ch/fr/2170.html.
}

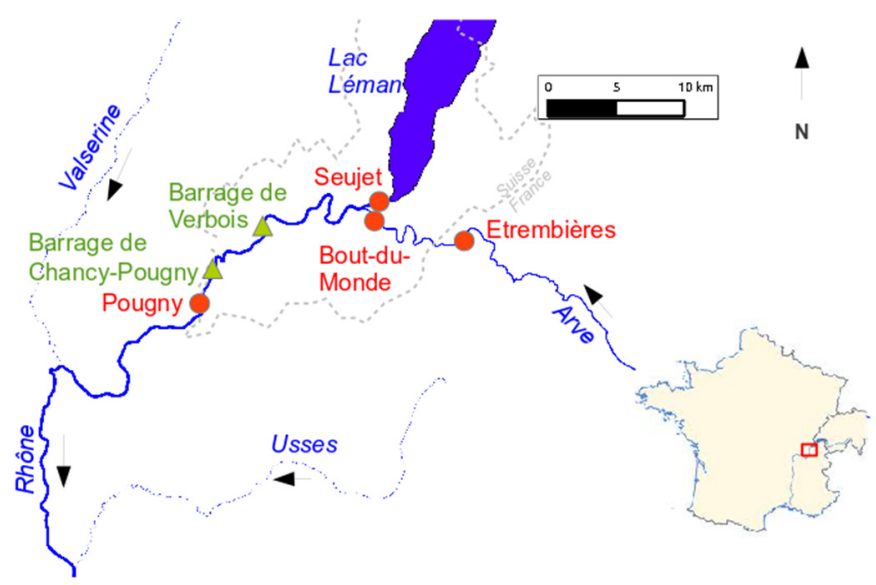

Fig. 1. Localisation de la station turbidimétrique sur l'Arve, à Genève-Bout-du-Monde, et du réseau hydrographique du secteur avec les stations débitmétriques (points rouges).

environ un quart du module du Rhône au niveau du quartier de Genève de la Jonction. Les débits instantanés maximaux pour les crues biennale, quinquennale et décennale sont respectivement de 487,593 et $654 \mathrm{~m}^{3} / \mathrm{s}$. La moitié du bassin versant se situe audessus de $1360 \mathrm{~m}$ d'altitude et des glaciers recouvrent en permanence environ $5 \%$ de la surface. Le régime hydrologique de l'Arve est soumis à la fonte des neiges à partir du printemps et jusqu'au début de l'automne (Bravard, 1987). L'Arve transporte surtout de fines particules détritiques qui s'accumulent en grande partie dans la retenue du barrage de Verbois, premier barrage sur le Rhône à l'aval de la confluence avec l'Arve. Lorsque l'Arve est en crue, il est habituel d'observer une zone de quatre à cinq kilomètres à l'aval de la confluence le long de laquelle les eaux turbides de l'Arve ne se sont pas encore mélangées aux eaux claires du Rhône provenant du lac Léman. Ce mélange est jugé effectif au niveau de la passerelle de Loëx, soit 4,7 km en aval de la Jonction.

Depuis mars 2012, une station de mesure turbidimétrique sur l'Arve à Genève au lieu-dit Bout-du-Monde (Fig. 1) est opérée par Irstea et les SIG dans le cadre de l'Observatoire des sédiments du Rhône. Cette station est située à environ $4 \mathrm{~km}$ en amont de la confluence de l'Arve avec le Rhône, elle-même située à seulement deux kilomètres en aval de l'embouchure du Lac Léman. Turbidimètre et échantillonneur automatique sont hébergés dans le local de la station débitmétrique de l'OFEV exploitée depuis 1924.

\subsection{Données recueillies}

Les enregistrements de débit moyen horaire sont disponibles pour l'Arve à Genève Bout-du-Monde de 1974 à 2017 (OFEV), pour le Rhône au Seujet en sortie du Léman (OFEV) et sur le Rhône à Pougny (CNR) en aval des barrages de Verbois et Chancy-Pougny. Nous disposons également des débits moyens journaliers de l'Arve à Genève Bout-du-Monde depuis 1904 (OFEV) et moyens mensuels de 1905 à 1911 à Etrembières (Service des Grandes Forces Hydrauliques), un peu plus en amont, en France.

Sur l'Arve à Genève Bout-du-Monde, les concentrations en MES ont été obtenues par prélèvements réguliers effectués par 


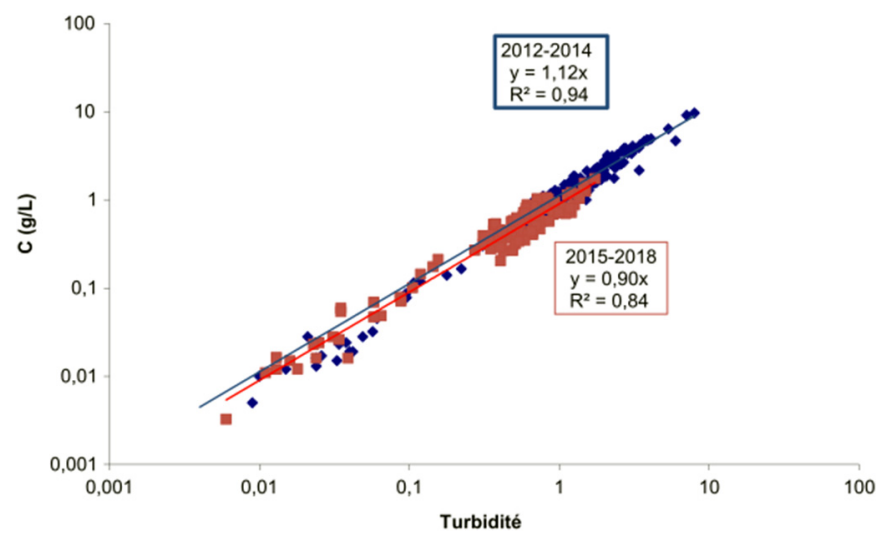

Fig. 2. Relations de calibration entre la concentration MES (g/L) et la turbidité (unités constructeur) établie entre mars 2012 et décembre 2014 (bleu) et entre janvier 2015 et février 2018 (rouge) à la station Genève Bout-du-Monde sur l'Arve.

l'OFEV de 1965 à 2003. Pour la période 1993-2003, les heures de prélèvement sont comprises entre 7 et $9 \mathrm{~h}$ (TU), avec une majorité des prélèvements réalisés à $7 \mathrm{~h}$ (TU). Faute d'indication de l'heure de prélèvement pour la période 1965-1993, nous avons considéré que les prélèvements étaient effectués à $7 \mathrm{~h}$ (TU). Pour la période 2003-2012, quelques prélèvements ponctuels sont disponibles.

Depuis la mise en service de la station turbidimétrique par Irstea et les SIG, la turbidité horaire a été calibrée à l'aide de prélèvements réalisés en régime de base et en crue, et la concentration en MES a été établie par filtration-pesée selon la méthode de la norme NF EN 872 (AFNOR, 2005). Le capteur de turbidité (sonde Hach-Lange Solitax 0-150 g/L) ayant été changé fin 2014, deux relations de calibration sont présentées sur la figure 2. Ces relations peuvent également dépendre de la qualité des particules transportées et notamment de leur taille (Thollet et al., 2013; Armijos et al., 2017). Des mesures de granulométrie sont ainsi menées pour évaluer l'effet d'un changement de taille des particules transportées en crue sur la relation de calibration.

Les variations annuelles de stock sédimentaire volumique de la retenue du barrage de Verbois sont estimées depuis 2004 par les SIG au moyen d'un modèle 3D construit sur des relevés bathymétriques réalisés une fois tous les trois ans. Nous disposons également de mesure de la granulométrie des dépôts et des MES prélevées manuellement en surface et à l'aide d'un piège à particules dans le Rhône à Pougny durant les chasses de barrage de 2012 (Launay, 2014).

\subsection{Erreur liée à un échantillonnage discontinu des concentrations en MES}

Le régime hydrologique de l'Arve est de type nival, avec un maximum en juin-juillet, et un minimum en février (Fig. 3). La période de basses eaux entre les mois de septembre et février est caractérisée par un débit moyen plus faible et par des épisodes de crue correspondant à des fortes pluies ou des orages. La période de hautes eaux des mois de mars à août correspond à la fonte des neiges. Elle est caractérisée par peu

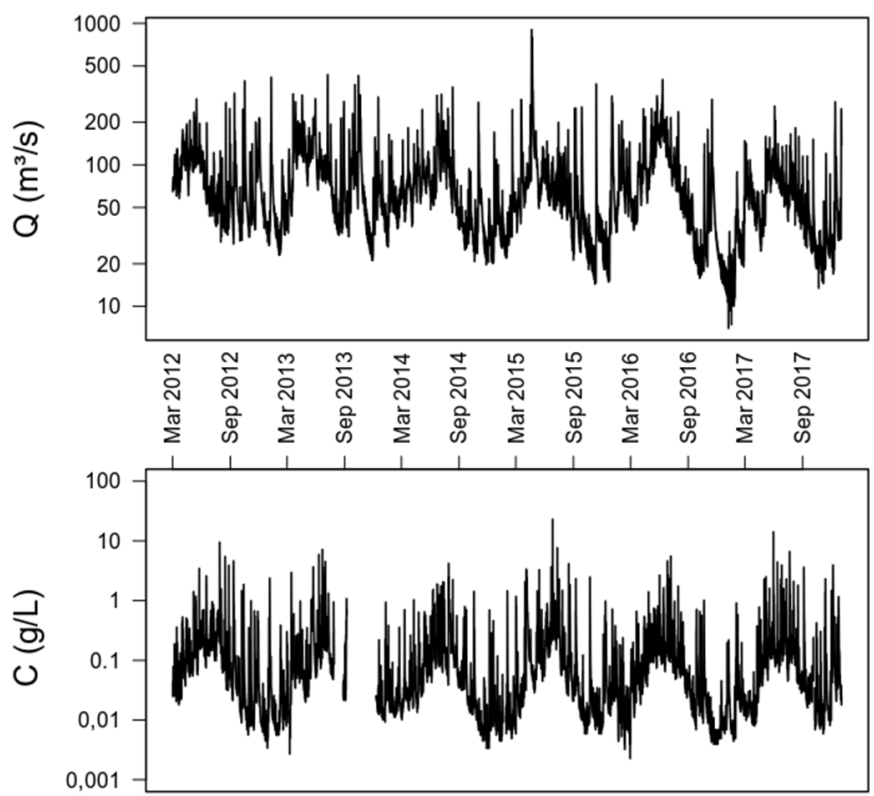

Fig. 3. Chroniques de débit (OFEV) et de concentration en MES (OSR) de mars 2012 à décembre 2017 à la station Genève-Bout-duMonde sur l'Arve ${ }^{14}$.

d'épisodes de crue importants mais par un débit moyen supérieur à celui de la période de pluie.

La représentativité d'une concentration échantillonnée à une heure donnée de la journée a été évaluée en établissant les statistiques des écarts de la concentration moyenne horaire de chaque heure de la journée à la concentration moyenne journalière (Fig. 4). Les résultats confirment que la période de fonte est marquée par un cycle journalier lié aux apports amont, tandis que la période de pluie ne présente pas de tendance cyclique significative au regard des fortes dispersions des écarts quelle que soit l'heure de prélèvement. Pendant la période de fonte, la concentration prélevée à $7 \mathrm{~h}$ présente une surestimation systématique de la moyenne journalière de 20 et de $15 \%$ pour la période de pluie.

\subsection{Méthodes de calcul du flux de MES}

Le flux de MES cumulé sur une période $P$ est calculé par l'équation suivante:

$$
\phi_{P}=\int_{P} Q(t) C(t) d t=\sum_{P} Q_{i} C_{i} \Delta t_{i},
$$

avec $\phi_{P}(\mathrm{~kg})$ le flux sur la période $P, Q\left(\mathrm{~m}^{3} / \mathrm{s}\right)$ le débit, $C(\mathrm{~g} / \mathrm{L})$ la concentration en MES et $\Delta t(\mathrm{~s})$ le pas de temps de mesure.

Dans le cas de l'Arve, le débit et la concentration peuvent varier d'un facteur deux à trois au cours de la journée et les crues durent de quelques heures à quelques jours. Un pas de temps horaire est donc le plus adapté pour étudier la dynamique du transport de MES et calculer des flux. La concentration en MES est mesurée à pas de temps horaire seulement depuis le début de l'année 2012, tandis que seules des données discontinues sont disponibles pour les années antérieures. 

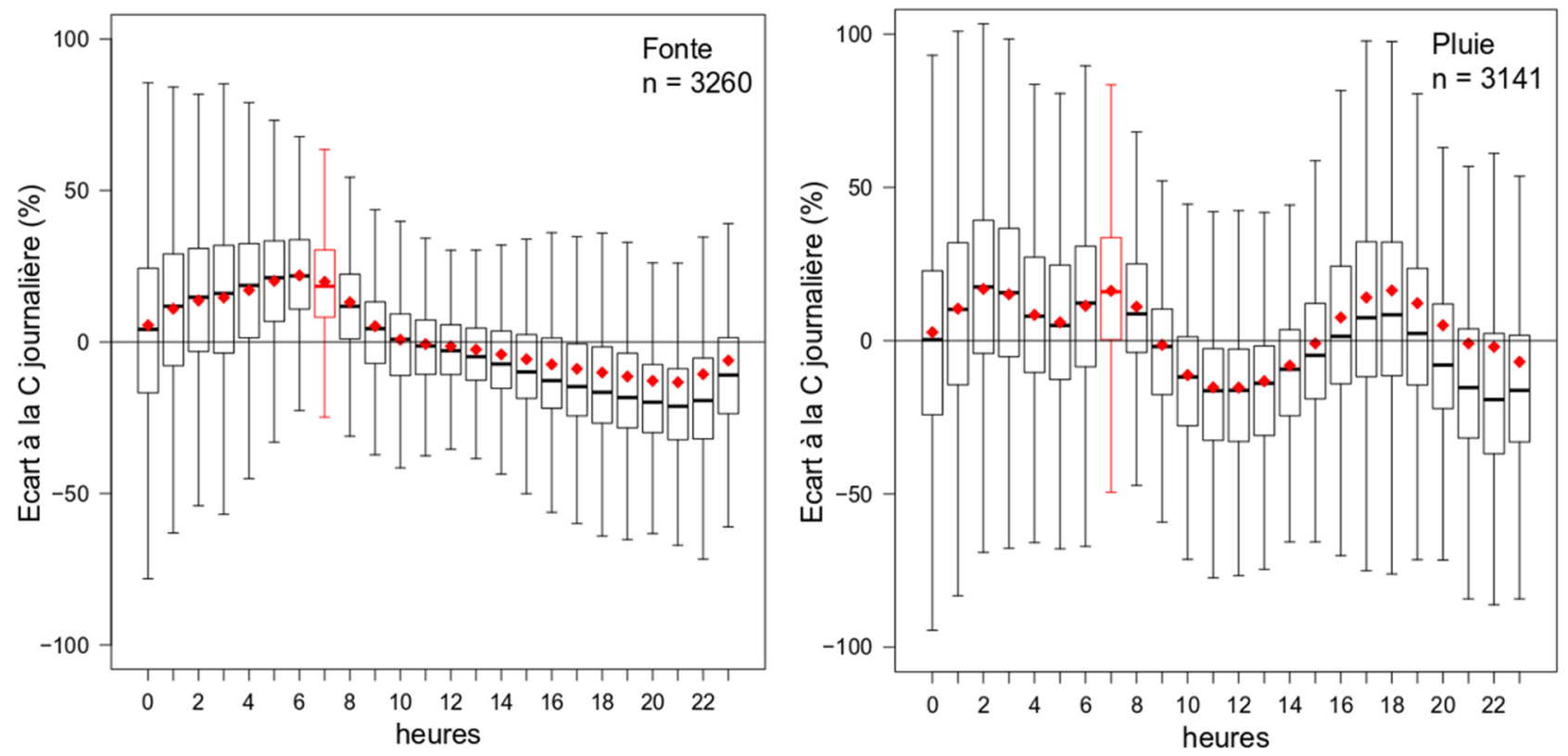

Fig. 4. Écarts des concentrations en MES moyennes horaires à la concentration moyenne journalière pendant la période de fonte (à gauche) et pendant la période de pluie (à droite). Les boîtes à moustache présentent médiane, quantiles 25 et $75 \%$, min/max et moyenne (point rouge). La mesure à $7 \mathrm{~h}$ est indiquée par une boîte à moustaches rouge. Les mesures utilisées correspondent à celles de la Figure 3 .

Dans ce travail, trois méthodes (décrites ci-après) sont utilisées pour calculer les flux en fonction des données de concentration en MES disponibles :

- pour les données continues, les flux sont calculés directement dans l'application $\mathrm{BDOH}^{3}$ (Branger et al., 2014);

- pour les données discontinues, les flux sont calculés par deux méthodes:

- la méthode des débits classés employée par l'OFEV pour son réseau de suivi des flux de MES (Grasso, 2003),

- la méthode de la courbe de tarage sédimentaire à deux segments.

\subsubsection{Méthode de calcul $\mathrm{BDOH}$}

Depuis 2012, les données de concentration en MES sont mesurées avec une périodicité de 10 minutes et moyennées sur $1 \mathrm{~h}$. Les flux sont calculés à pas de temps horaire, directement dans la base de données BDOH, puis sommés sur l'année, par application de l'équation (1) aux débits et concentrations en MES moyens horaires:

$$
\phi_{\mathrm{an}}=\sum_{\mathrm{an}} Q_{h} C_{h} \times 3600
$$

avec $\phi_{\text {an }}$ le flux cumulé annuel $(\mathrm{kg}), Q_{h}$ le débit moyen horaire $\left(\mathrm{m}^{3} / \mathrm{s}\right)$ et $C_{h}$ la concentration moyenne horaire $\left(\mathrm{kg} / \mathrm{m}^{3}\right)$.

Les lacunes sont comblées à l'aide de la méthode de la courbe de tarage sédimentaire ( $c f$. Sect. 2.4.3).

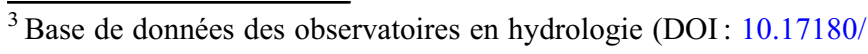
OBS.OSR) : https://bdoh.irstea.fr/.
}

\subsubsection{Méthode des débits classés}

Entre 1965 et 2003, les données de concentrations en MES ont été mesurées en moyenne tous les 5 jours à $7 \mathrm{~h}$. L'OFEV préconise d'utiliser la méthode des débits classés, détaillée par Grasso (2003). Cette méthode repose sur l'analyse de la courbe des débits classés, qui représente la fréquence de dépassement des valeurs de débit sur la période considérée. Les débits utilisés par l'OFEV sont les débits moyens journaliers. Les couples débit-concentration sont triés selon les débits décroissants et répartis selon 29 classes de débit de tailles différentes. Le flux moyen calculé pour chacune de ces classes de débit dépend uniquement des mesures de concentration appartenant à cette classe. Les flux moyens par classe sont ensuite pondérés par la taille de la classe de débit et sommés pour obtenir le flux sur la période considérée. Le flux $\phi_{p}$ en tonnes pour la période $P$ est donc calculé selon l'équation suivante :

$$
\phi_{p}=p \frac{k}{100} \sum_{i=1}^{N} \frac{\Delta_{i}}{\mathrm{M}_{\mathrm{i}}}\left[\sum_{\mathrm{j}=1}^{\mathrm{M}_{\mathrm{i}}} \mathrm{Q}_{\mathrm{j}} \mathrm{C}_{\mathrm{j}}\right]_{i}
$$

avec $p$ le nombre de jours ou d'heures de la période $P, k$ le facteur de conversion des $\mathrm{kg} / \mathrm{s}$ en $\mathrm{t} / \mathrm{j}$ ou en $\mathrm{t} / \mathrm{h}, N$ le nombre de classes, $\Delta_{i}$ la largeur de la classe $i$ (en pourcentage d'occurrence), $M_{i}$ le nombre d'échantillons de la classe $i, C_{j}$ la concentration en MES du jour ou de l'heure $j\left(\mathrm{en} \mathrm{kg} / \mathrm{m}^{3}\right)$ et $Q_{j}$ le débit du jour ou de l'heure $j\left(\mathrm{en}^{3} / \mathrm{s}\right)$.

\subsubsection{Méthode de la courbe de tarage sédimentaire}

L'établissement d'une courbe de tarage pour reproduire la dynamique sédimentaire d'un cours d'eau à partir des débits est couramment effectuée (Horowitz, 2003; Asselman, 1999; Walling et Webb, 1985). L'hypothèse sous-jacente est que 
l'évolution de la concentration en MES est liée simplement aux variations de débit. La chronique de concentration est reconstituée à partir d'une relation empirique entre le débit et la concentration de MES. La relation la plus couramment recherchée est une fonction puissance entre le débit et la concentration en MES. Le flux devient alors :

$$
\phi_{P}=\sum_{i} a Q_{i}^{b+1}
$$

Toutefois, Grasso (2003) souligne la faiblesse de ce type de relation qui s'ajuste mal pour les fortes valeurs, faiblement représentées. L'extrapolation de la relation $\mathrm{C}(\mathrm{Q})$ pour des débits supérieurs à ceux échantillonnés peut donc conduire à une importante erreur sur les concentrations en MES. Sadaoui et al. (2016) et Poulier et al. (2019) ont montré l'intérêt d'utiliser des relations $\mathrm{C}(\mathrm{Q})$ à deux segments pour reconstituer des chroniques de concentration en MES à partir du débit. Cette méthode a été utilisée dans ces deux articles sur le Rhône et ses principaux affluents, mais l'Arve n'y est pas présenté.

La relation à deux segments utilisée dans cette étude (Fig. 5) a été établie selon la méthode présentée dans Poulier et al. (2019). Elle a été utilisée pour calculer les flux de MES entre 1973 et 2012 et pour combler les lacunes dans les données continues de 2012 à 2018 .

\section{3 Évaluation des flux moyens de particules fines exportés par l'Arve}

Les flux annuels de particules fines ont été calculés sur les années hydrologiques allant du $1^{\text {er }}$ septembre au 31 août de l'année civile suivante. Les concentrations en MES moyennes sont calculées en divisant les flux cumulés de MES par les volumes d'eau écoulés sur la même période de temps.

\subsection{Comparaison des méthodes de calcul}

Grasso (2003) a comparé les flux de MES obtenus par la méthode des débits classés et par la courbe de tarage sédimentaire, aux flux obtenus par la mesure de la concentration en MES chaque jour à la même heure pendant un an à la station de Lütshine-Gsteig en Suisse. En faisant l'hypothèse que cette concentration est représentative de la concentration moyenne journalière, il a établi un flux de référence en faisant le produit de la concentration en MES et du débit journalier et en sommant sur l'année. Il a ainsi conclu que la méthode des débits classés donnait un flux plus proche du flux de référence et que la méthode de la courbe de tarage sous-estimait de $62 \%$ la valeur du flux.

Nous avons appliqué la même démarche au jeu de données horaires mesuré entre 2012 et 2017 à Genève-Bout-du-Monde sur l'Arve. Pour reproduire la fréquence d'échantillonnage du suivi OFEV entre 1965 et 2003, nous avons sous-échantillonné le jeu de données en gardant une valeur tous les 5 jours à $7 \mathrm{~h} \mathrm{du}$ matin. Cet échantillonnage permet d'obtenir cinq jeux de données auxquels nous avons appliqués la méthode des débits classés. La courbe de tarage sédimentaire testée est celle obtenue à partir de l'ensemble des données disponibles entre 1973 et 2017.

Les flux de MES obtenus par la mesure en continu et par les deux méthodes de calcul sont présentés sur la figure 6. Les

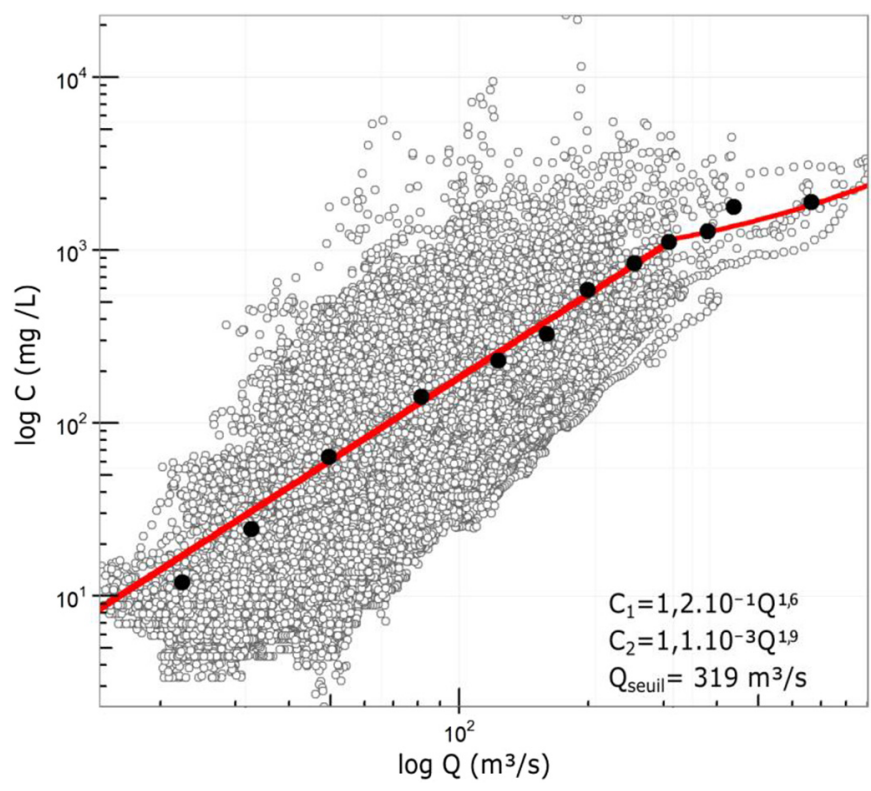

Fig. 5. Relation à deux segments entre le débit $Q$ et la concentration en MES (C) établie selon la méthode décrite dans Poulier et al. (2019) et inspirée par Sadaoui et al. (2016) pour la station de l'Arve à Genève Bout-du-Monde (données de 2000-2017). Les points noirs sont des moyennes par classe de débit (limites en fréquences : 0,1, 0,25, 0,5, 1 , $2,5,10,20,50,75$ et $90 \%$ ). Le calage des 5 paramètres de la relation à 2 segments de loi puissance a été effectué avec l'estimateur bayésien BaRatin (Le Coz et al., 2014).

enveloppes d'erreur associées sont de $10 \%$ pour le flux obtenu par la mesure en continu, de $20 \%$ pour la courbe de tarage sédimentaire, et les valeurs minimales et maximales obtenues avec les cinq échantillons pour la méthode des débits classés.

Cette étude montre que les flux obtenus par les deux méthodes de calcul sont généralement assez proches des flux mesurés. Cependant, les flux obtenus par la méthode des débits classés sont très sensibles à l'échantillonnage. Pour les cinq années étudiées, le ratio entre l'écart-type et la moyenne des flux obtenus avec les cinq échantillons différents varie de 12 à $41 \%$. En effet, les pics de crue de l'Arve passant en seulement quelques heures, la fréquence d'échantillonnage de 5 jours peut amener à rater complètement les crues d'une année, et obtenir une représentation très partielle de l'hydrologie du cours d'eau. La courbe de tarage sédimentaire établie à partir du jeu de données continues (pas de temps horaire) de concentration permet donc de mieux représenter la dynamique sédimentaire, à partir du moment où la gamme de concentration en MES et de débit utilisés pour l'établir couvre les crues, comme c'est le cas ici.

Pour les années hydrologiques 1974-1975 à 2002-2003 (Fig. 7), les flux de MES calculés par la méthode des débits classés ont une moyenne interannuelle de 0,73 Mt. Avec la courbe de tarage sédimentaire, nous obtenons un flux moyen de 0,74 Mt. Les flux de MES moyens interannuels sont donc équivalents. Néanmoins, pour la méthode des débits classés, l'année 1992-1993 constitue une exception (Fig. 7). Le flux moyen est extrêmement élevé (plus de $3 \mathrm{Mt}$ ); si l'on rapporte cette valeur au flux d'eau moyen annuel, on obtient une concentration en MES supérieure à $2 \mathrm{~g} / \mathrm{L}$ en moyenne sur 


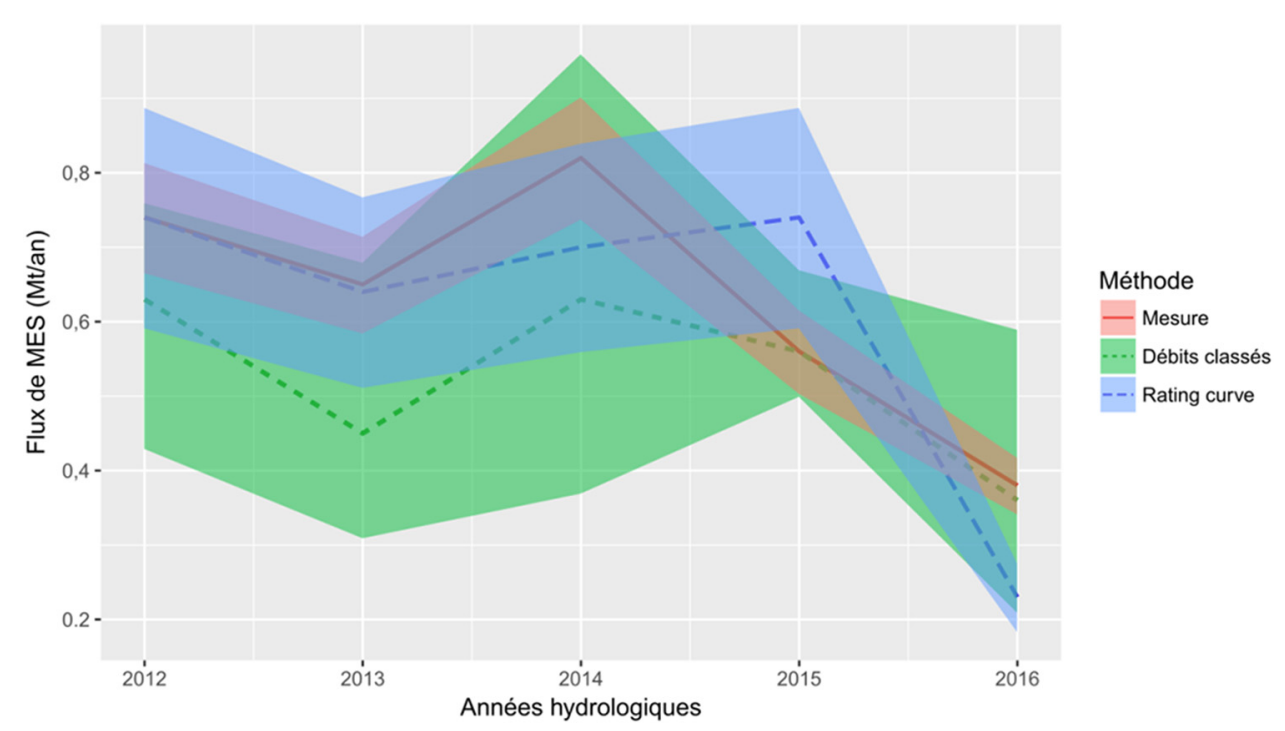

Fig. 6. Flux de MES de l'Arve à la station Genève Bout-du-Monde obtenu pour les années hydrologiques (de septembre à août) 2012 à 2016 par la mesure en continu, la méthode des débits classés et la méthode de la courbe de tarage sédimentaire à deux segments. Les zones de couleur représentent les incertitudes associées aux flux (voir texte).

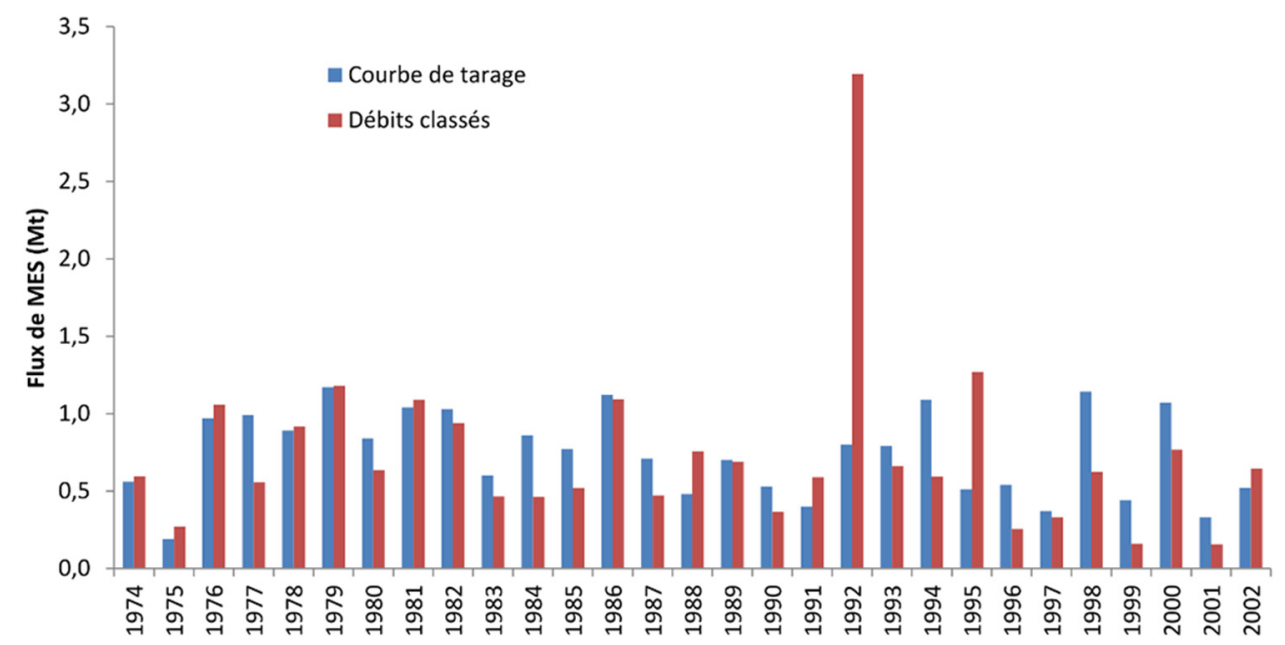

Fig. 7. Flux de MES de l'Arve entre 1974 et 2002 (années hydrologiques de septembre à août) avec les méthodes des débits classés et de la courbe de tarage sédimentaire à deux segments.

l'année, ce qui est irréaliste. La méthode des débits classés montre ici ses limites. En 1992, 196 valeurs de concentrations en MES ont été enregistrées dont la plus élevée atteint $30 \mathrm{~g} / \mathrm{L}$ (la seconde plus forte valeur est de $13 \mathrm{~g} / \mathrm{L}$ ). Cette valeur correspond au 4ème plus fort débit de l'année. Le flux associé à cette classe est donc élevé. Avec un échantillonnage plus fin, un plus grand nombre de crues auraient été enregistrées, ce qui diminuerait le poids associé aux fortes valeurs de débit. Une seule très forte valeur rend ainsi le flux calculé par cette méthode invraisemblable.

Dans la suite de cette étude, nous avons utilisé la méthode de la courbe de tarage sédimentaire pour calculer les flux de MES pour la période 1973-2012, pour laquelle les débits horaires sont disponibles. Pour la période 1965-1973, les résultats de l'OFEV obtenus par la méthode des débits classés ont été conservés.

\subsection{Flux moyens annuels entre 1965 et 2017}

À partir des données de MES et de débit des suivis OFEV et Irstea, nous avons calculé les débits et flux de MES annuels sur la période 1965 à 2017. Les flux de MES et les concentrations moyennes sont représentés sur la figure 8 et les valeurs sont données en Annexe 1. Le flux d'eau moyen interannuel est de $2,39 \mathrm{Gm}^{3} /$ an (soit un débit moyen interannuel de $76 \mathrm{~m}^{3} / \mathrm{s}$ ), dont $65 \%$ en moyenne en période de fonte. Les écoulements maximaux ont eu lieu en 1965-1966 (3,24 Gm³ $\left./ \mathrm{an}, 103 \mathrm{~m}^{3} / \mathrm{s}\right)$ et en 1994-1995 $\left(3,21 \mathrm{Gm}^{3} / \mathrm{an}, 102 \mathrm{~m}^{3} / \mathrm{s}\right)$. Ces deux années sont marquées par des tempêtes à l'automne et des événements neigeux importants de janvier à mars, d'après Météo France. Les années les plus sèches ont eu lieu en 1975-1976 et en 2010-2011 (1,55-1,56 Gm³/an, $\left.49 \mathrm{~m}^{3} / \mathrm{s}\right)$, deux années de forts épisodes de sécheresse en Europe, avec très peu de 


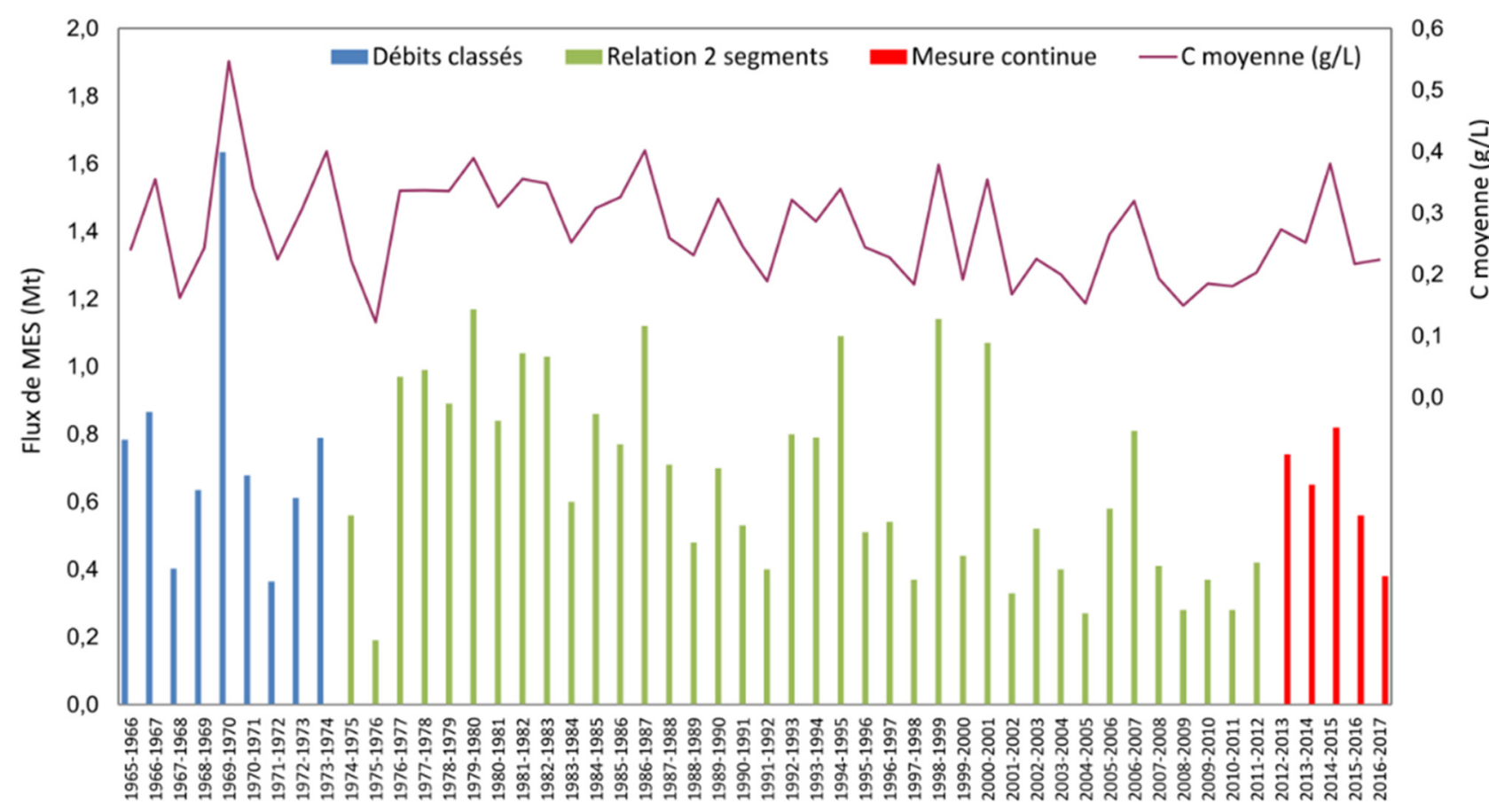

Fig. 8. Évolution des flux et des concentrations moyennes des MES de l'Arve entre 1965 et 2016 (années hydrologiques de septembre à août).

précipitations à l'automne et durant l'hiver. Les débits mesurés reproduisent bien ces observations météorologiques.

Les années de fort débit moyen annuel correspondent généralement à des années de flux annuel de MES élevé et inversement. La concentration de MES moyenne interannuelle est de $274 \mathrm{mg} / \mathrm{L}$. L'année 1969-1970 se distingue avec une concentration de MES moyenne de $546 \mathrm{mg} / \mathrm{L}$. En effet, le suivi de l'OFEV présente une longue période sans mesure entre novembre 1969 et janvier 1970. Le jeu de données est donc particulièrement peu documenté en période de basses eaux. Par ailleurs, des événements de crues moyennes se sont produits pendant tout l'été. La méthode des débits classés utilisée ici conduit probablement à une surestimation de la concentration moyenne et du flux annuel de MES puisque seuls les débits des jours associés à un prélèvement de MES sont pris en considération dans le calcul.

Le flux moyen interannuel de MES entre 1965 et 2017 est de 0,68 Mt/an avec un écart-type de $0,29 \mathrm{Mt} / \mathrm{an}$, ce qui est cohérent avec le flux moyen mesuré par la station turbidimétrique entre 2012 et 2017 (0,63 Mt/an). La proportion de flux de MES en période de fonte varie entre 32 et $95 \%$ avec une moyenne de $71 \%$. La variabilité temporelle du flux moyen annuel est fortement corrélée à la part de la fonte, qui représente la majorité du flux de MES.

\subsection{Retour sur les valeurs de flux de MES proposées dans la littérature}

Dans son remarquable travail de thèse, Baëff (1891) a mesuré quotidiennement les concentrations en MES et le débit de l'Arve pendant onze mois de l'année 1890 (le mois d'octobre manque). Des prélèvements de surface ont été effectués tous les jours entre une heure et deux heures de l'après-midi à Genève. Il en déduit un flux total de 0,61 Mt de MES (valeur incomplète reprise par Meybeck et al. (2003) pour leur comparatif de 60 stations du globe). Lugeon (1912) a complété ce résultat en estimant à $0,06 \mathrm{Mt}$ le flux de MES pour le mois d'octobre 1890 , d'où un total de de $0,67 \mathrm{Mt}$ pour l'année 1890. En se basant sur une étude des données pluviométriques enregistrées par l'Observatoire de Genève, il conclut que «l'année 1890 représente une période de charriage [sic] un peu au-dessous de la moyenne $»$. Il propose donc de retenir $0,70 \mathrm{Mt}$ comme valeur moyenne annuelle de flux de MES.

Collet (1916) a remis en question le flux de MES mesuré par Baëff (1891) en critiquant la méthode d'établissement des débits utilisée par celui-ci. Baëff a en effet calculé le débit en mesurant la vitesse superficielle de l'écoulement sur les bords et au centre de l'écoulement, et en multipliant la moyenne de ces trois valeurs par le coefficient 0,80. Collet (1916) estime que les débits calculés par cette méthode sont «largement sous-estimés ». Nous pensons que le coefficient de 0,80 utilisé par Baëff (1891) est en effet un peu faible. La valeur de coefficient d'intégration verticale usuellement utilisée pour ce calcul est de 0,86 et dans le cas de l'Arve, il pourrait même être porté à 0,91 ( $\mathrm{cf}$. norme ISO748, AFNOR (2009)). De plus, le poids donné aux vitesses proches des bords entraîne aussi une sous-estimation de la vitesse moyenne qui devrait être corrigée en augmentant le coefficient. Le débit calculé par Baëff (1891) pourrait donc être sous-estimé d'environ $10 \%$. Collet (1916) pointe notamment que la moyenne annuelle des débits obtenus par Baëff (1891) est de $54 \mathrm{~m}^{3} / \mathrm{s}$, alors que la moyenne des années 1904-1916 s'élève à $83 \mathrm{~m}^{3} / \mathrm{s}$. Les débits moyens interannuels mesurés par l'OFEV entre 1973 et $2013\left(76 \mathrm{~m}^{3} / \mathrm{s}\right)$ et par le service des grandes forces hydrauliques à Etrembières (Ministère de l'Agriculture, 1912) entre 1905 et $1911\left(72 \mathrm{~m}^{3} / \mathrm{s}\right)$ sont également plus élevés. Pour corriger les débits de Baëff (1891) pour l'année 1890, Collet (1916) utilise un jaugeage au flotteur réalisé le 27 mai 1903 par le 
Tableau 1. Caractéristiques hydro-sédimentaires de l'Arve à Genève comparées à celles d'autres bassins versants. Sources : [1] Meybeck et al., 2003 ; [2] Syvitski et al., 2005 ; [3] le présent article : Launay et al., 2019; [4] Baëff, 1891 ; [5] Poulier et al., 2019; [6] Thollet et al., 2018 ; [7] Camenen et al., 2018; [8] Némery et al., 2013.

\begin{tabular}{|c|c|c|c|c|c|c|c|c|}
\hline Bassins versants & Réf. & $\begin{array}{l}\text { Années } \\
\text { de suivi }\end{array}$ & $\begin{array}{l}\text { Surface } \\
\text { du bassin } \\
\left(\mathrm{km}^{2}\right)\end{array}$ & $\begin{array}{l}\text { Débit moyen } \\
\left(\mathrm{m}^{3} / \mathrm{s}\right)\end{array}$ & $\begin{array}{l}\text { Débit } \\
\text { spécifique } \\
\left(\mathrm{L} / \mathrm{s} / \mathrm{km}^{2}\right)\end{array}$ & $\begin{array}{l}\text { Concentration } \\
\text { moyenne }^{\mathrm{a}} \\
(\mathrm{g} / \mathrm{L})\end{array}$ & $\begin{array}{l}\text { Flux moyen } \\
\text { (Mt/an) }\end{array}$ & $\begin{array}{l}\text { Flux } \\
\text { spécifique } \\
\left(\mathrm{kg} / \mathrm{km}^{2} / \text { jour }\right)\end{array}$ \\
\hline $\begin{array}{l}60 \text { stations dans } \\
\text { le monde }\end{array}$ & 1 & Variable & 64 à $3.210^{6}$ & - & $0.1-55$ & $0.005-29$ & - & $10-5000$ \\
\hline Monde & 2 & Époque moderne & $1,0610^{8}$ & $1,2210^{6}$ & 12 & 0,327 & $12,610^{3}$ & 326 \\
\hline Europe & 2 & Époque moderne & $10^{7}$ & $8510^{3}$ & 8 & 0,254 & 680 & 186 \\
\hline $\begin{array}{l}\text { Low mountain } \\
1000-3000 \mathrm{~m}\end{array}$ & 2 & Époque moderne & $3610^{6}$ & $4,010^{5}$ & 11 & 0,375 & 4800 & 365 \\
\hline Arve à Genève & 3 & $1965-2017$ & 1976 & 75 & 40 & 0,288 & 0,68 & 943 \\
\hline Arve à Genève & 1,4 & 1890 sans octobre & 2079 & 58 & 28 & 0,335 & 0,61 & 820 \\
\hline Rhône à Jons & 5 & $2000-2016$ & 20058 & 576 & 29 & 0,034 & 0,62 & 85 \\
\hline $\begin{array}{l}\text { Arc au seuil de } \\
\text { Pontamafrey }\end{array}$ & 6,7 & 2011-2017 & 1506 & 15,5 & 10 & 1,254 & 0,613 & 1115 \\
\hline Isère à Grenoble Campus & 8 & 2006-2008 & 5570 & 200 & 36 & $0,206-0,365$ & $1,3-2,3$ & $639-1131$ \\
\hline Isère à Grenoble Campus & 6 & 2007-2016 & 5570 & 166 & 30 & 0,345 & 1,81 & 891 \\
\hline Isère à Beaumont-Monteux & 5 & $2000-2016$ & 11794 & 309 & 26 & 0,155 & 1,64 & 381 \\
\hline Durance à Bonpas & 5 & 2000-2016 & 14124 & 68 & 5 & 0,644 & 1,55 & 301 \\
\hline Rhône à Beaucaire & 5 & $2000-2016$ & 96363 & 1630 & 17 & 0,128 & 6,56 & 186 \\
\hline
\end{tabular}

${ }^{\text {a }}$ Concentration moyenne pondérée par les débits.

Service de l'Hydrographie nationale auquel il applique la méthode de calcul de Baëff. Il démontre ainsi le biais des résultats de Baëff (1891) et en tire une méthode de ré-estimation des débits de 1890. Collet (1916) précise que l'ingénieur Pesson chargé de cette ré-analyse a proposé une valeur corrigée de débit pour chaque jour de 1890 et obtient un débit moyen annuel de $75 \mathrm{~m}^{3} / \mathrm{s}$ et un flux de MES pour les onze mois de mesure de Baëff de $0,65 \mathrm{Mt}$. En ajoutant à cette valeur le flux d'octobre proposé par Lugeon (1912), on obtient un flux de MES de 0,71 Mt pour l'année 1890 , au lieu de 0,67 : en réalité, il ne s'agissait donc pas d'une « large sous-estimation » puisque la différence est estimée à seulement $-6 \%$.

Ce flux estimé pour 1890 est cohérent avec notre estimation du flux moyen interannuel de MES entre 1965 et 2017 (0,68 Mt/an avec un écart-type de 0,29 Mt/an). Il ne faut néanmoins pas oublier que les flux annuels de l'Arve sont très variables du fait des fortes crues, qui diffèrent beaucoup d'une année sur l'autre. En considérant l'exemple de la crue extrême du 2 au 4 octobre 1888, Baëff (1891) lui-même calcule que «pendant la journée du 3 octobre 1888 , où le débit était $1136 \mathrm{~m}^{3}$ à la seconde, il s'écoula en un seul jour $98150400 \mathrm{~m}^{3}$. En portant la charge d'alluvions à $3 \mathrm{~kg} / \mathrm{m}^{3}$, qui est évidemment trop faible, on arrive au chiffre fantastique de 294450 tonnes de matières en suspension renfermées dans l'eau ». Ainsi, il est probable que cette crue ait pu transporter en seulement $24 \mathrm{~h}$ l'équivalent de la moitié du flux moyen interannuel.

Le tableau 1 permet de remettre les caractéristiques hydrosédimentaires de l'Arve à Genève en perspective avec des évaluations sur d'autres bassins versants, ainsi que les moyennes mondiales et européennes. L'Arve se distingue par un débit spécifique très élevé, néanmoins proche de celui de l'Isère et sans doute de l'Arc en Maurienne dont les données sont modifiées par les dérivations d'eau vers l'Isère (qui augmentent artificiellement la concentration moyenne en baissant le débit moyen). La concentration moyenne de l'Arve est dans la moyenne de sa catégorie (Isère à Grenoble-Campus, montagnes entre 1000 et $3000 \mathrm{~m}$ ), et les moyennes d'Europe ou du Monde, mais elle est bien moins élevée que celles de la Durance ou de l'Arc par exemple (cette dernière étant artificialisée comme indiqué précédemment). Le flux moyen annuel de l'Arve est légèrement supérieur à celui du Rhône à Jons, ce qui en fait l'apport principal du Haut-Rhône, l'excédent s'expliquant par le stockage de matériau dans les retenues. Le flux moyen de l'Arve représente environ $10 \% \mathrm{du}$ flux total du Rhône à la mer, évalué à Beaucaire. Enfin, l'Arve présente un flux spécifique élevé (proche de celui de l'Isère à Grenoble-Campus) en raison de son débit spécifique élevé.

\section{Estimation du flux moyen de suspension grossière}

\subsection{Informations historiques sur le flux de suspension hétérogène}

Le 4 août 1915, pour un débit de $262 \mathrm{~m}^{3} / \mathrm{s}$, Collet et Boissier (Collet, 1925) ont effectué 32 prélèvements distribués dans la section de l'Arve à Vessy pour mesurer le transport de matières en suspension. Collet (1925) en conclut que le flux de MES calculé à partir des seules mesures en surface est inférieur au flux de sédiments en suspension passant sur l'ensemble de la section de mesure d'un facteur 2,129 (qu'on peut raisonnablement arrondir à 2,1). Durant toute l'année 1915, Collet et Boissier ont effectué un «suivi détaillé » des MES (prélèvements de surface) et ont obtenu un 
flux annuel de MES de 1,71 Mt. Ils en déduisent que le flux de suspension totale réellement transporté par l'Arve est de 3,64 Mt pour l'année 1915.

Collet (1925) souligne que l'année 1915 est une année d'abondance (débits importants) et que cette valeur est donc un maximum. En effet, en 1915, d'après les données journalières de l'OFEV, le débit moyen annuel était de $104 \mathrm{~m}^{3} / \mathrm{s}$ (soit $3,28 \mathrm{Gm}^{3}$ ) et l'année a été marquée par d'importantes crues, dont cinq événements avec un débit journalier supérieur à $250 \mathrm{~m}^{3} / \mathrm{s}$ pendant la période de fonte. Le débit annuel de 1915 est supérieur au débit moyen sur la période 1965-2017 $\left(76 \pm 14 \mathrm{~m}^{3} / \mathrm{s}\right)$ et excède d'environ $2 \%$ ceux des années 1965 et 1994, les deux années les plus abondantes de la période. Avec des parts de fonte de 61 et $59 \%$, ces années sont donc comparables avec l'année 1915 au niveau de l'hydrologie (Annexe 1). Pourtant, les flux de MES de 1965 et de 1994 ne représentent respectivement que 45 et $63 \%$ du flux de MES obtenu par Collet (1925) pour 1915. Collet (1925) ne donne pas d'informations sur le protocole de prélèvement suivi ni sur la méthode de calcul de flux employée, précisant simplement qu'un «suivi détaillé » a été mené. Il est possible que les prélèvements aient été effectués principalement en crue ce qui conduirait à une surestimation des flux obtenus sur l'année. L'heure de mesure pourrait également introduire un biais dans le calcul de flux, mais qui ne saurait expliquer un tel écart, comme discuté précédemment.

En appliquant le coefficient 2,129 au flux de MES (fines) calculé pour l'année 1890, Collet (1925) obtient un flux de suspension totale (grossiers inclus) de 1,51 Mt; considérant que le débit de l'année 1890 est inférieur à la moyenne des débits mesurés entre 1904 et 1916, il pense que ce flux de suspension totale est également inférieur à la moyenne. Sur la base des données du Service des grandes forces hydrauliques et de l'OFEV, nous pensons que c'est plutôt la moyenne des débits utilisée par Collet entre 1904 et $1916\left(83 \mathrm{~m}^{3} / \mathrm{s}\right)$ qui est un peu surestimée, l'année 1890 présentant un débit moyen annuel qui se situe dans la moyenne des débits mesurés sur l'Arve et non dans la gamme basse.

Cette étude rétrospective sur l'hydrologie et l'estimation des flux de MES dans l'Arve a permis d'expliquer l'origine probable des valeurs de flux de «MES» estimées pour l'Arve dans l'Étude Globale Rhône (SOGREAH, 2000) qui fait à ce jour référence. La fourchette proposée est de 1,0 à 3,6 Mt/an, basée sur les valeurs de la littérature et donc probablement de l'étude de Collet (1925) citée par Pardé (1942). Or, comme nous l'avons expliqué, Collet (1925) fonde ses estimations non pas sur des flux de MES calculés à partir de prélèvements en surface mais sur des flux de suspension totale (sables en suspension hétérogène inclus), en appliquant un coefficient correctif de 2,129 aux premiers pour estimer les seconds.

L'application d'un simple facteur 2,129 pour englober le flux de sables et en déduire un flux total comme proposé par Collet (1925), paraît extrêmement hasardeuse, comme le soulignait déjà Pardé (1942). En l'absence de mesures récentes, fréquentes et directes de ce flux de suspensions grossières, la meilleure information à notre disposition est le suivi du comblement et de la granulométrie des sédiments de la retenue de Verbois, qui reçoit un flux de sédiments quasi exclusivement issus de l'Arve, puisque le Rhône en sortant du Léman transporte une charge sédimentaire négligeable.

\subsection{Bilan du flux de suspension totale autour de la retenue de Verbois}

Le comblement moyen annuel de la retenue de Verbois est estimé à $360000 \mathrm{~m}^{3} / \mathrm{an}$ par les SIG qui réalisent depuis 2004 des mesures bathymétriques une fois tous les trois ans. Ces dépôts présentent une granulométrie fine; par conséquent, ils ne sont pas uniquement constitués par le piégeage de suspension de sables, mais aussi pour bonne partie de suspension fine (MES). En 2009, des carottes de sédiment ont été prélevées dans quatre zones de l'amont à l'aval de la retenue de Verbois, dans des dépôts remobilisables par les chasses (Institut Forel, 2009). L'analyse granulométrique des matériaux indique un fort gradient longitudinal, avec un taux de particules de diamètre inférieur à $63 \mu \mathrm{m}$ égal à $9,49,77$ et $84 \%$ pour les quatre zones (notées A, B, C, D) de l'amont vers l'aval. Les zones $\mathrm{C}$ et $\mathrm{D}$ sont celles où s'effectue préférentiellement la sédimentation. La fraction argileuse $(<2 \mu \mathrm{m})$ est quasiment négligeable partout.

Ces mesures sont cohérentes avec les analyses granulométriques qui ont été effectuées par Irstea dans le cadre de l'OSR en juin 2012 sur des sédiments remobilisés par les opérations de chasse du barrage de Verbois (Launay, 2014). Les prélèvements ont été effectués à Pougny soit manuellement en surface (MES), soit à l'aide d'un piège à sédiments placé en berge sur un escalier immergé (incluant de la suspension hétérogène). Dans les deux types d'échantillons, les modes lognormaux suivants ont été obtenus en décomposant les distributions granulométriques (Masson et al., 2018) pour la méthode de décomposition granulométrique): sable fin $(d=85-107 \mu \mathrm{m})$, limon $(d=15-63 \mu \mathrm{m})$, limon fin/argile $(d=3-8 \mu \mathrm{m})$. Les proportions de ces fractions diffèrent cependant pour les deux types de prélèvement. Le sable est absent des prélèvements manuels en surface, mais il représente de 50 à $60 \%$ des particules du piège à sédiments, exposé à la suspension hétérogène. La fraction la plus fine ne représente que $10 \%$ des particules pour les deux types d'échantillons.

Convertir les volumes de dépôt en masses de sédiment sec nécessite de connaître la densité des matériaux en place. Les densités de matériau sec des carottes prélevées par l'institut Forel (2009) présentent une décroissance de l'amont vers l'aval de la retenue (zones A, B, C, D) : 1400, 1315, 1365, $1200 \mathrm{~kg} / \mathrm{m}^{3}$, avec une moyenne de $1320 \mathrm{~kg} / \mathrm{m}^{3}$. Ces valeurs sont cohérentes avec celles classiquement indiquées pour des sédiments de retenue immergés en permanence, notamment USDI (2006) (Tab. 2). En considérant les valeurs minimale, maximale et moyenne des densités observées, la masse de sédiment stockée annuellement dans la retenue du barrage de Verbois correspondant à l'envasement de $360000 \mathrm{~m}^{3} / \mathrm{an}$ serait donc comprise entre 0,43 et $0,50 \mathrm{Mt}$, avec une valeur centrale de $0,47 \mathrm{Mt} / \mathrm{an}$.

En faisant l'hypothèse (pas si irréaliste) que la totalité du sable issu de l'Arve est piégé dans la retenue de Verbois, et en considérant (à partir des relevés granulométriques des zones $\mathrm{C}$ et D) que les $3 / 4$ de ces $0,45 \mathrm{Mt} /$ an sont constitués de MES fines, on aboutit à un apport de $0,12 \mathrm{Mt} / \mathrm{an}$ de sables par l'Arve. De même, 0,35 Mt/an de MES fines, soit grossièrement la moitié des apports moyens de l'Arve, seraient arrêtées dans la retenue de Verbois. De telles estimations du flux de sables et du taux de dépôt dans la retenue de Verbois restent très incertaines et appellent des observations plus détaillées pour être affinées. 
Tableau 2. Densité des sédiments en fonction de leur classe granulométrique (d'après USDI, 2006).

\begin{tabular}{lc}
\hline & Densité $\left(\mathrm{kg} / \mathrm{m}^{3}\right)$ \\
\hline Argiles $(d<4 \mu \mathrm{m})$ & 416 \\
Limons $(4<d<62 \mu \mathrm{m})$ & 1200 \\
Sables $(d>62 \mu \mathrm{m})$ & 1500 \\
\hline
\end{tabular}

\section{Conclusion}

Cette étude a permis de réévaluer les apports moyens en suspension fine de l'Arve, principal contributeur au flux de matières en suspension du Rhône entre le lac Léman et Lyon. En croisant les données historiques avec les flux de MES de référence pour les années 2012 à 2017 issus du suivi turbidimétrique en continu à la station de Genève Bout-duMonde de l'Observatoire des sédiments du Rhône, les deux méthodes de calcul des flux mises en œuvre par le Service hydrologique national suisse ont été évaluées pour les périodes avec des mesures discontinues des MES : la méthode des débits classés et la courbe de tarage sédimentaire à deux segments. Il a également été possible d'évaluer l'erreur systématique sur le flux de MES potentiellement induite par l'effet de l'échantillonnage temporel du suivi conduit par l'OFEV entre 1965 et 2003 (prélèvement vers $7 \mathrm{~h}$ tous les 5 jours en moyenne) : pendant la période de fonte, la concentration prélevée à $7 \mathrm{~h}$ présente une surestimation systématique de la moyenne journalière de 20 et de $15 \%$ pour la période de pluie.

En combinant au mieux ces deux méthodes de calcul et le suivi turbidimétrique des années récentes, le flux moyen interannuel entre 1965 et 2017 a été estimé à $0,68 \mathrm{Mt} / \mathrm{an}$ avec un écart-type de 0,29 Mt/an. Cette valeur est cohérente avec le flux moyen mesuré à partir des mesures en continu par la station turbidimétrique entre 2012 et $2017(0,63 \mathrm{Mt})$ et avec un suivi historique pour l'année $1890(0,71 \mathrm{Mt})$. Cette valeur moyenne ne doit pas faire oublier la forte variabilité temporelle des flux de MES, avec des valeurs moyennes annuelles observées de 0,2 à $1,2 \mathrm{Mt}$, et un flux estimé d'au moins $0,3 \mathrm{Mt}$ pour la seule crue du 3 octobre 1888 .

Notre estimation du flux moyen de l'Arve est nettement inférieure à l'estimation précédemment proposée par l'étude globale Rhône (2000), comprise entre 1,0 et 3,6 Mt/an, mais qui ajoute vraisemblablement au flux de suspension fine homogène (MES) le flux de sables et limons grossiers en suspension hétérogène, qui demeure très mal connu. Selon la problématique étudiée, il est capital de ne pas confondre les deux flux - MES et suspension totale - qui correspondent à des populations sédimentaires très différentes en termes de granulométrie, affinité pour les contaminants hydrophobes, et comportement mécanique (cohésion, transport, dépôt et resuspension). Le facteur 2,129 appliqué au flux de suspension fine pour calculer le flux de suspension totale est issu d'expériences menées pendant une année de fort débit en 1915 (Collet, 1925) et son application est très discutable.

D'après les données disponibles sur le comblement de la retenue de Verbois et la granulométrie des particules déposées et remobilisées pendant la chasse de juin 2012, le flux de sables est vraisemblablement beaucoup plus faible, de l'ordre de
0,12 Mt/an, et la moitié environ des flux de MES fines serait piégée dans la retenue. Ces estimations restent beaucoup plus incertaines que les valeurs du flux moyen de MES fines. Le flux sédimentaire de l'Arve en suspension graduée (sables, limons grossiers) et charriage (graviers) mériterait de faire l'objet de nouvelles observations et évaluations, notamment en documentant les distributions granulométriques et en réalisant des prélèvements distribués au fond et dans la section et des mesures hydro-acoustiques lors d'événements mobilisateurs.

Remerciements. Ce travail a été mené grâce à l'Observatoire des Sédiments du Rhône (OSR), programme de recherche pluri-partenaires financé dans le cadre du Plan Rhône par le Fonds Européen de Développement Economique et Régional (FEDER), l'Agence de l'eau Rhône Méditerranée Corse, CNR, EDF et les Régions Auvergne-Rhône-Alpes, PACA et Occitanie. La thèse de Marina Launay (2010-2013) a été financée par la Région Rhône-Alpes (ARC Environnement). Nous remercions vivement l'OFEV pour l'accès aux données de débit, de concentration et de flux de MES de la station de Genève Bout-du-Monde.

\section{Références}

AFNOR. 2005. Norme NF EN 872. Qualité de l'eau - Dosage des matières en suspension - Méthode par filtration sur filtre en fibres de verre.

AFNOR. 2009. Norme NF EN ISO 748. Hydrométrie - Mesurage du débit des liquides dans les canaux découverts au moyen de moulinets ou de flotteurs.

Armijos E, Crave A, Espinoza R, et al. 2017. Measuring and modeling vertical gradients in suspended sediments in the Solimões/Amazon River. Hydrol Process 31(3): 654-667.

Asselman NEM. 1999. Suspended sediment dynamics in a large drainage basin: the River Rhine. Hydrol Process 13: 1437-1450.

Baëff B. 1891. Les eaux de l'Arve : recherches de géologie expérimentale sur l'érosion et le transport dans les rivières torrentielles ayant des affluents glaciaires. Thèse de doctorat : Univ. Genève.

Branger F, Thollet F, Crochemore M, et al. 2014. Le projet Base de données pour les observatoires en hydrologie: un outil pour la bancarisation, la gestion et la mise à disposition des données issues des observatoires hydrologiques de long terme à Irstea. La Houille Blanche 1: 33-38.

Bravard JP. 1987. Le Rhône, col. L'Homme et la nature, La Manufacture, $451 \mathrm{p}$.

Camenen B, Archambault J, Thollet F, Rousseau C, Némery J. 2018. Spatial and temporal dynamics of suspended sediment fluxes in an alpine river: the Arc and Isère rivers, France, AGU Fall meeting, Washington DC, USA.

Collet LW. 1916. I Le charriage des alluvions dans certains cours d'eau de la Suisse. Berne : Annales du Service Suisse des Eaux n ${ }^{\circ}$, $257 \mathrm{p}$.

Collet LW. 1925. Les lacs, leur mode de formation, leurs eaux, leur destin. Éléments d'hydrogéologie. Paris : Librairie d'Octave Doin, et Gaston Doin, éditeur, 320 p.

Grasso DA. 2003. Charge de sédiments en suspension. Comparaison entre deux méthodes de calcul. GWA (OFEV), 898-905.

Guertault L, Camenen B, Paquier A, Peteuil C. 2018. A onedimensional process-based approach to study reservoir sediment dynamics during management operations. Earth Surf Process Landforms 43(2): 373-386. 
Horowitz A. 2003. An evaluation of sediment rating curves for estimating suspended sediment concentration for subsequent flux calculation. Hydrol Process 17: 3387-3409.

Launay M. 2014. Flux de matières en suspension, de mercure et de PCB particulaires dans le Rhône, du Léman à la Méditerranée. France : PhD, Université Lyon 1, $466 \mathrm{p}$.

Le Bescond C, Thollet F, Le Coz J, et al. 2018. Des flux d'eau aux flux de matières en suspension et de contaminants associés: gestion d'un réseau de stations hydro-sédimentaires sur le Rhône. $L a$ Houille Blanche 3: 63-70.

Le Coz J, Renard B, Bonnifait L, Branger F, Le Boursicaud R. 2014. Combining hydraulic knowledge and uncertain gaugings in the estimation of hydrometric rating curves: a Bayesian approach. $J$ Hydrol 509: 573-587.

Lugeon M. 1912. Étude géologique sur le projet de barrage du HautRhône français à Génissiat, Mémoires de la Société géologique de France, 4ème sér., t. II, mémoire $n^{\circ} 8$.

Masson M, Angot H, Le Bescond C, et al. 2018. Sampling of suspended particulate matter using particle traps in the Rhône River: relevance and representativeness for the monitoring of contaminants. Sci Total Environ 637-638: 538-549.

Meybeck M, Laroche L, Dürr H, Syvitski JPM. 2003. Global variability of daily-suspended solids and their load in rivers. Global Planetary Change 39: 65-93.

Ministère de l'Agriculture. 1912. Service des grandes forces hydrauliques dans la région des Alpes. Tome V - Résultats des études et travaux à la fin de 1911.

Némery J, Mano V, Coynel A, et al. 2013. Carbon and suspended sediment transport in an impounded alpine river (Isère, France). Hydrol Process 27(17): 2498-2508.

Pardé. 1942, réédition 2004. Quelques nouveautés sur le régime du Rhône. coll. Géocarrefour, 139 p.
Poulier M, Launay M, Le Bescond C, Thollet F, Coquery M, Le Coz J. 2019. Combining flux monitoring and data reconstruction to establish annual budgets of suspended particulate matter, mercury and PCB in the Rhône River from Lake Geneva to the Mediterranean Sea. Sci Total Environ 658: 457-473.

Sadaoui M, Ludwig W, Bourrin F, Raimbault P. 2016. Controls, budgets and variability of riverine sediment fluxes to the Gulf of Lions (NW Mediterranean Sea). J Hydrol 540: $1002-1015$.

SOGREAH. 2000. Étude globale pour une stratégie de réduction des risques dus aux crues du Rhône. Étude du transport solide. $1^{\text {re }}$ étape - Diagnostic de l'état actuel - Calculs de transport solide, Institution interdépartementale des bassins Rhône-Saône (IRS), $48 \mathrm{p}$.

Syvitski JPM, Vörösmarty JV, Kettner AJ, Green P. 2005. Impacts of humans on the load of terrestrial sediments to the global coastal ocean. Science 308: 376-380.

Thollet F, Le Coz J, Antoine G, et al. 2013. Influence de la granulométrie des particules sur la mesure de turbidité et le calcul des flux sédimentaires. Conférence SHF Hydrométrie 2013, 15-16, Paris, France.

Thollet F, Camenen B, Le Coz J, Némery J, Rousseau C. 2018. Suivi du transport sédimentaire sur les rivières Arvan, Arc et Isère. https://dx.doi.org/10.17180/OBS.ARC-ISERE.

US Dept of the Interior. 2006. Erosion and sedimentation manual. Denver, Colorado, USA: U.S. Dept. of the Interior, Bureau of Reclamation, Technical Service Center, Sedimentation and River Hydraulics Group, 602 p.

Walling D, Webb B. 1985. Estimating the discharge of contaminants to coastal waters by rivers: some cautionary comments. Marine Pollution Bull 16: 488-492. 
Annexe 1 Flux d'eau et de MES annuels calculés à partir des suivis de l'OFEV et de l'OSR sur la période 1965-2016, en années hydrologiques de septembre à août (valeurs maximales en rouge, valeurs minimales en bleu).

\begin{tabular}{|c|c|c|c|c|c|c|c|}
\hline & \multicolumn{3}{|c|}{ Eau } & \multicolumn{3}{|c|}{ Matières en suspension } & \multirow{11}{*}{ 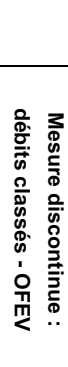 } \\
\hline & Débit $\left(\mathrm{m}^{3} / \mathrm{s}\right)$ & Flux $\left(\mathrm{Gm}^{3}\right)$ & $\%$ Fonte & Flux (Mt) & C moyenne (g/L) & $\%$ Fonte & \\
\hline 1965-1966 & 103,3 & 3,26 & 61 & 0,78 & 0,241 & 65 & \\
\hline 1966-1967 & 77,3 & 2,44 & 69 & 0,87 & 0,355 & 93 & \\
\hline 1967-1968 & 78,6 & 2,48 & 67 & 0,40 & 0,162 & 75 & \\
\hline 1968-1969 & 82,7 & 2,61 & 60 & 0,63 & 0,243 & 72 & \\
\hline $1969-1970$ & 94,7 & 2,99 & 74 & 1,63 & 0,546 & 86 & \\
\hline $1970-1971$ & 63,1 & 1,99 & 66 & 0,68 & 0,341 & 87 & \\
\hline 1971-1972 & 51,3 & 1,62 & 70 & 0,36 & 0,224 & 92 & \\
\hline $1972-1973$ & 63,1 & 1,99 & 71 & 0,61 & 0,307 & 68 & \\
\hline $1973-1974$ & 62,4 & 1,97 & 65 & 0,79 & 0,400 & 65 & \\
\hline 1974-1975 & 79,5 & 2,51 & 59 & 0,56 & 0,223 & 66 & \multirow{38}{*}{ 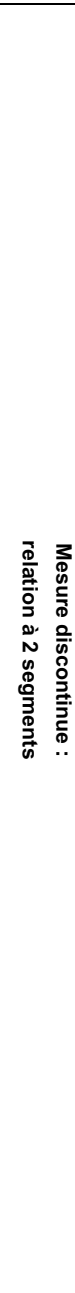 } \\
\hline $1975-1976$ & 49,4 & 1,56 & 52 & 0,19 & 0,122 & 32 & \\
\hline $1976-1977$ & 91,6 & 2,89 & 66 & 0,97 & 0,336 & 72 & \\
\hline $1977-1978$ & 93,2 & 2,94 & 69 & 0,99 & 0,337 & 83 & \\
\hline $1978-1979$ & 84,0 & 2,65 & 68 & 0,89 & 0,335 & 73 & \\
\hline $1979-1980$ & 95,4 & 3,01 & 66 & 1,17 & 0,389 & 78 & \\
\hline $1980-1981$ & 85,9 & 2,71 & 66 & 0,84 & 0,309 & 79 & \\
\hline $1981-1982$ & 92,8 & 2,93 & 57 & 1,04 & 0,355 & 54 & \\
\hline $1982-1983$ & 93,8 & 2,96 & 63 & 1,03 & 0,348 & 66 & \\
\hline 1983-1984 & 75,4 & 2,38 & 62 & 0,60 & 0,252 & 62 & \\
\hline 1984-1985 & 88,4 & 2,79 & 64 & 0,86 & 0,308 & 72 & \\
\hline $1985-1986$ & 74,8 & 2,36 & 78 & 0,77 & 0,326 & 95 & \\
\hline $1986-1987$ & 88,4 & 2,79 & 75 & 1,12 & 0,401 & 92 & \\
\hline 1987-1988 & 86,8 & 2,74 & 66 & 0,71 & 0,259 & 80 & \\
\hline $1988-1989$ & 65,6 & 2,07 & 61 & 0,48 & 0,232 & 46 & \\
\hline $1989-1990$ & 68,8 & 2,17 & 70 & 0,70 & 0,323 & 69 & \\
\hline $1990-1991$ & 68,4 & 2,16 & 56 & 0,53 & 0,246 & 51 & \\
\hline 1991-1992 & 67,2 & 2,12 & 65 & 0,40 & 0,188 & 75 & \\
\hline $1992-1993$ & 78,9 & 2,49 & 52 & 0,80 & 0,321 & 40 & \\
\hline 1993-1994 & 87,5 & 2,76 & 56 & 0,79 & 0,286 & 51 & \\
\hline 1994-1995 & 101,7 & 3,21 & 59 & 1,09 & 0,339 & 61 & \\
\hline $1995-1996$ & 66,2 & 2,09 & 65 & 0,51 & 0,244 & 75 & \\
\hline $1996-1997$ & 75,4 & 2,38 & 64 & 0,54 & 0,227 & 78 & \\
\hline 1997-1998 & 63,7 & 2,01 & 60 & 0,37 & 0,184 & 54 & \\
\hline 1998-1999 & 95,4 & 3,01 & 68 & 1,14 & 0,378 & 79 & \\
\hline $1999-2000$ & 72,6 & 2,29 & 60 & 0,44 & 0,192 & 61 & \\
\hline $2000-2001$ & 95,7 & 3,02 & 69 & 1,07 & 0,354 & 85 & \\
\hline 2001-2002 & 62,4 & 1,97 & 67 & 0,33 & 0,168 & 82 & \\
\hline 2002-2003 & 73,2 & 2,31 & 51 & 0,52 & 0,225 & 35 & \\
\hline 2003-2004 & 63,4 & 2,00 & 61 & 0,40 & 0,200 & 55 & \\
\hline 2004-2005 & 56,1 & 1,77 & 68 & 0,27 & 0,153 & 78 & \\
\hline $2005-2006$ & 69,4 & 2,19 & 78 & 0,58 & 0,265 & 95 & \\
\hline 2006-2007 & 80,5 & 2,54 & 70 & 0,81 & 0,319 & 89 & \\
\hline 2007-2008 & 67,2 & 2,12 & 70 & 0,41 & 0,194 & 85 & \\
\hline 2008-2009 & 59,6 & 1,88 & 61 & 0,28 & 0,149 & 57 & \\
\hline 2009-2010 & 63,4 & 2,00 & 67 & 0,37 & 0,185 & 78 & \\
\hline $2010-2011$ & 49,1 & 1,55 & 60 & 0,28 & 0,180 & 57 & \\
\hline 2011-2012 & 65,6 & 2,07 & 68 & 0,42 & 0,203 & 71 & \\
\hline 2012-2013 & 85,9 & 2,71 & 61 & 0,74 & 0,273 & 64 & \multirow{5}{*}{ 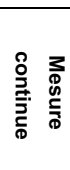 } \\
\hline 2013-2014 & 81,8 & 2,58 & 55 & 0,65 & 0,252 & 54 & \\
\hline 2014-2015 & 68,4 & 2,16 & 69 & 0,82 & 0,380 & 93 & \\
\hline 2015-2016 & 81,8 & 2,58 & 66 & 0,56 & 0,217 & 70 & \\
\hline 2016-2017 & 53,9 & 1,70 & 69 & 0,38 & 0,224 & 87 & \\
\hline Moyenne & 75,7 & 2,39 & 65 & 0,68 & 0,273 & 71 & \\
\hline Ecart-type & 13,9 & 0,44 & 6,1 & 0,29 & 0,083 & 15,6 & \\
\hline
\end{tabular}

Citation de l'article : Launay M, Le Coz J, Diouf S, Camenen B, Thollet F, Coquery M. 2019. Réévaluation des apports moyens de matières en suspension de l'Arve au Rhône. La Houille Blanche 2: 89-100 